\title{
Baseline visual acuity strongly predicts visual acuity gain in patients with diabetic macular edema following anti-vascular endothelial growth factor treatment across trials
}

\author{
This article was published in the following Dove Press journal: \\ Clinical Ophthalmology \\ 14 June 2016 \\ Number of times this article has been viewed
}

\begin{abstract}
Pravin U Dugel ${ }^{1,2}$
Jost Hillenkamp ${ }^{3}$

Sobha Sivaprasad ${ }^{4,5}$

Jessica Vögeler ${ }^{6}$

Marie-Catherine Mousseau ${ }^{7}$

Andreas Wenzel ${ }^{8}$

Philippe Margaron ${ }^{8}$

Ron Hashmonay ${ }^{8}$

Pascale Massin ${ }^{9}$

'Retinal Consultants of Arizona LTD, Retinal Research Institute LLC, Phoenix, AZ, ${ }^{2}$ USC Eye Institute, Keck School of Medicine, University of Southern California, Los Angeles, CA, USA; ${ }^{3}$ Department of Ophthalmology, Julius-Maximilians University, Würzburg, Germany; ${ }^{4} \mathrm{NIHR}$ Biomedical Research Centre, Moorfields Eye Hospital, London, ${ }^{5}$ King's College Hospital, London, UK; ${ }^{6}$ Novartis Pharma GmbH, Nürnberg, Germany; ${ }^{7}$ Novartis Ireland Limited, Dublin, Ireland; ${ }^{8}$ Novartis Pharma AG, Basel, Switzerland; ' Department of Ophthalmology, Hôpital Lariboisière, Assistance Publique des Hôpitaux de Paris, Université Paris Diderot, Paris, France
\end{abstract}

Correspondence: Pravin U Dugel Retinal Consultants of Arizona LTD, I I0I E. Missouri Avenue Phoenix, AZ 85014, USA

Tel + I 602222222 I

Email pdugel@gmail.com
Objective: This study was designed to evaluate the correlation of baseline visual acuity (VA) with VA outcome in response to anti-vascular endothelial growth factor (VEGF) in diabetic macular edema using a retrospective analysis of nine clinical trials. The result will help assess the relevance of VA gain comparisons across trials.

Methods: A correlation analysis was performed between mean baseline VA and VA gain at month 12 for 1,616 diabetic macular edema patients across nine randomized clinical trials (RESOLVE, RISE, RIDE, RESTORE, RETAIN, DRCR.net Protocol I, DA VINCI, VIVID, VISTA) with anti-VEGF treatment regimens ranibizumab $0.5 \mathrm{mg}$ and aflibercept $2 \mathrm{mg}$.

Results: The mean baseline VA ranged from 56.9 to 64.8 Early Treatment Diabetic Retinopathy Study (ETDRS) letters. The mean VA gain at month 12 ranged from 6.8 to 13.1 ETDRS letters across trials. There was a strong inverse correlation between mean baseline VA and VA gain at month 12 ( $r=-0.85)$. The mean VA at 12 months plateaued at $\sim 0$ (68.5-73.0) ETDRS letters (20/40 Snellen VA equivalent) for the anti-VEGF treatment groups from all trials, regardless of dosing regimens and agents.

Conclusion: Cross-trial comparisons based on changes in best-corrected visual acuity should be done cautiously and only after adjusting for best-corrected visual acuity at baseline. Furthermore, the total VA afforded by treatment appears to be subject to a plateau effect, which warrants further exploration.

Keywords: aflibercept, anti-vascular endothelial growth factor, best-corrected visual acuity, cross-trial comparison, diabetic macular edema, ranibizumab

\section{Introduction}

Diabetic retinopathy (DR) is the most common microvascular complication of diabetes $^{1,2}$ and a leading cause of visual impairment and blindness. DR can lead to diabetic macular edema (DME), which affects $\sim 30 \%$ of patients who have had diabetes for at least 20 years $^{3}$ and is responsible for much of the vision loss due to DR. Vascular endothelial growth factor (VEGF) inhibitors, administered by intravitreal injection, have become established as part of the de facto standard of care in DME. As our understanding of the profiles of anti-VEGF agents in ophthalmology was refined, there has been a growing interest in exploring different regimens and drugs to maximize efficacy while minimizing burden on patients and health care systems.

Prospective, randomized clinical trials have addressed the efficacy and safety of different types of anti-VEGF in the treatment of DME, including pegaptanib, ranibizumab, 
bevacizumab, and aflibercept. These clinical trials have shown wide variations in efficacy in terms of visual acuity (VA) gains in patients with DME, not only among trials with different anti-VEGF agents or regimens but also between trials with similar agent and regimen. For instance, in the RESTORE trial in DME, ranibizumab $0.5 \mathrm{mg}$ administered according to a pro re nata $(\mathrm{PRN})$ regimen (plus laser) resulted in 12-month gain of only 6.4 letters, ${ }^{4}$ in contrast to a gain of nine letters with ranibizumab $0.5 \mathrm{mg}$ PRN (plus laser) in Diabetic Retinopathy Clinical Research Network Protocol I study (Protocol I). ${ }^{5}$

Such differences in apparent efficacy warrant closer attention. Comparing the performance of anti-VEGF agents involves cross-trial comparisons, with not only different compounds and administration regimens but also different trial populations. DR is known to be a variable disease that is dependent on both local and systemic factors, which are very difficult to control. In addition, due to varying inclusion and exclusion criteria, the mean best-corrected visual acuity (BCVA) at baseline differs substantially between trials, which may distort comparisons across trials that use change in BCVA as an efficacy end point.

The objective of the present study was to determine the contribution of baseline BCVA to assessments of drug efficacy in DME in an analysis of nine clinical trials of different anti-VEGF agents and treatment regimens.

\section{Methods}

A cross-DME trial comparison was conducted on data from nine clinical trials of anti-VEGF agents in DME, such as Protocol I, ${ }^{5}$ RESOLVE, ${ }^{6}$ RESTORE, ${ }^{4}$ RISE, ${ }^{7}$ RIDE, ${ }^{7}$ DA VINCI, ${ }^{8}$ VIVID,${ }^{9}$ VISTA,${ }^{9}$ and RETAIN. ${ }^{10}$ All studies were conducted in compliance with the tenets of the Declaration of Helsinki. Approvals were obtained from the independent Ethics Committee or Institutional Review boards and all patients provided written informed consent before enrollment into the trials. Ethical approval was not sought from any IRB for the present analysis, as it only uses study-level data publicly available from these previously approved studies. The selected studies were all Phase II or Phase III randomized controlled trials of ranibizumab or aflibercept using mean BCVA change at 12 months (or 24 months for RISE and RIDE) as an efficacy end point. It should be noted that BCVA gains for RISE and RIDE at 24 months are very similar to gains observed at 12 months, as illustrated by the plateau curve displaying the time course of VA gains in these two trials. ${ }^{7}$ DA VINCI, VIVID, and VISTA assessed the effect of a maintenance regimen of aflibercept every four weeks (q4), every eight weeks (q8), or PRN (DA VINCI) on mean BCVA. RESOLVE, RISE, RIDE, Protocol I, RESTORE, and RETAIN evaluated
BCVA outcomes with a maintenance regimen of ranibizumab administered monthly, PRN, and/or treat and extend depending on the trial. In some of these trials, monthly injections were performed prior to maintenance therapy (Table 1).

\section{Inclusion/exclusion criteria}

Inclusion and exclusion criteria varied between studies, notably in terms of baseline VA. In RETAIN and RESTORE, DME patients were eligible if they had a BCVA between 39 and 78 letters, both inclusive, based on Early Treatment Diabetic Retinopathy Study (ETDRS)-like VA testing charts (approximate Snellen equivalent 20/160 and 20/32); in RESOLVE, BCVA letter scores required for inclusion were between 39 and 73 ETDRS letters (approximate Snellen equivalent 20/160-20/40); in Protocol I, BCVA scores required for inclusion were 24-78 ETDRS letters (Snellen equivalent 20/320-20/32); in RISE and RIDE, DA VINCI, VIVID, and VISTA, BCVA scores required for inclusion were 24-73 ETDRS letters (Snellen equivalent 20/320-20/40).

Inclusion criteria for retinal thickness measured on time domain optical coherence tomography in the central subfield, when present, also varied from $\geq 250 \mu \mathrm{m}$ (DA VINCI, Protocol I) to $\geq 275 \mu \mathrm{m}$ (RISE and RIDE) and $\geq 300 \mu \mathrm{m}$ (RETAIN [if BCVA >73], RESOLVE, VIVID). Stable diabetes was usually required, with glycated hemoglobin $(\mathrm{HbA} 1 \mathrm{c}) \leq 12 \%$ in RESOLVE, RETAIN, RISE and RIDE, and VIVID; HbA $1 \mathrm{c} \leq 10 \%$ in RESTORE; or in the opinion of the investigator (VISTA, DA VINCI). Generally, patients who received antiangiogenic drugs within 3 months prior to the study (RESTORE, RISE and RIDE, RETAIN, DA VINCI, VISTA, and VIVID) or any DME treatment within 4 months (Protocol I) were excluded. In RESOLVE, all patients who received any previous/current intravitreal or sub-Tenon drug delivery and/or participated in a clinical trial involving antiangiogenic drugs were excluded. Active proliferative DR was part of the exclusion criteria in RESOLVE, RESTORE, RETAIN, VISTA, and VIVID.

\section{Statistical analysis}

The mean baseline BCVA values and mean BCVA gains were taken from each published paper for the 14 arms that used aflibercept $2 \mathrm{mg}$ or ranibizumab $0.5 \mathrm{mg}$ (the doses licensed in many countries including the European Union) as monotherapy (or with deferred laser, the most analogous to monotherapy; Table 1) and summed to provide absolute BCVA levels at 12 months.

In addition, a simple linear regression model was used, and the correlation (Pearson's $r$ ) between mean baseline BCVA and mean BCVA gain at 12 months was determined using data from all anti-VEGF treatment arms of the nine trials. 


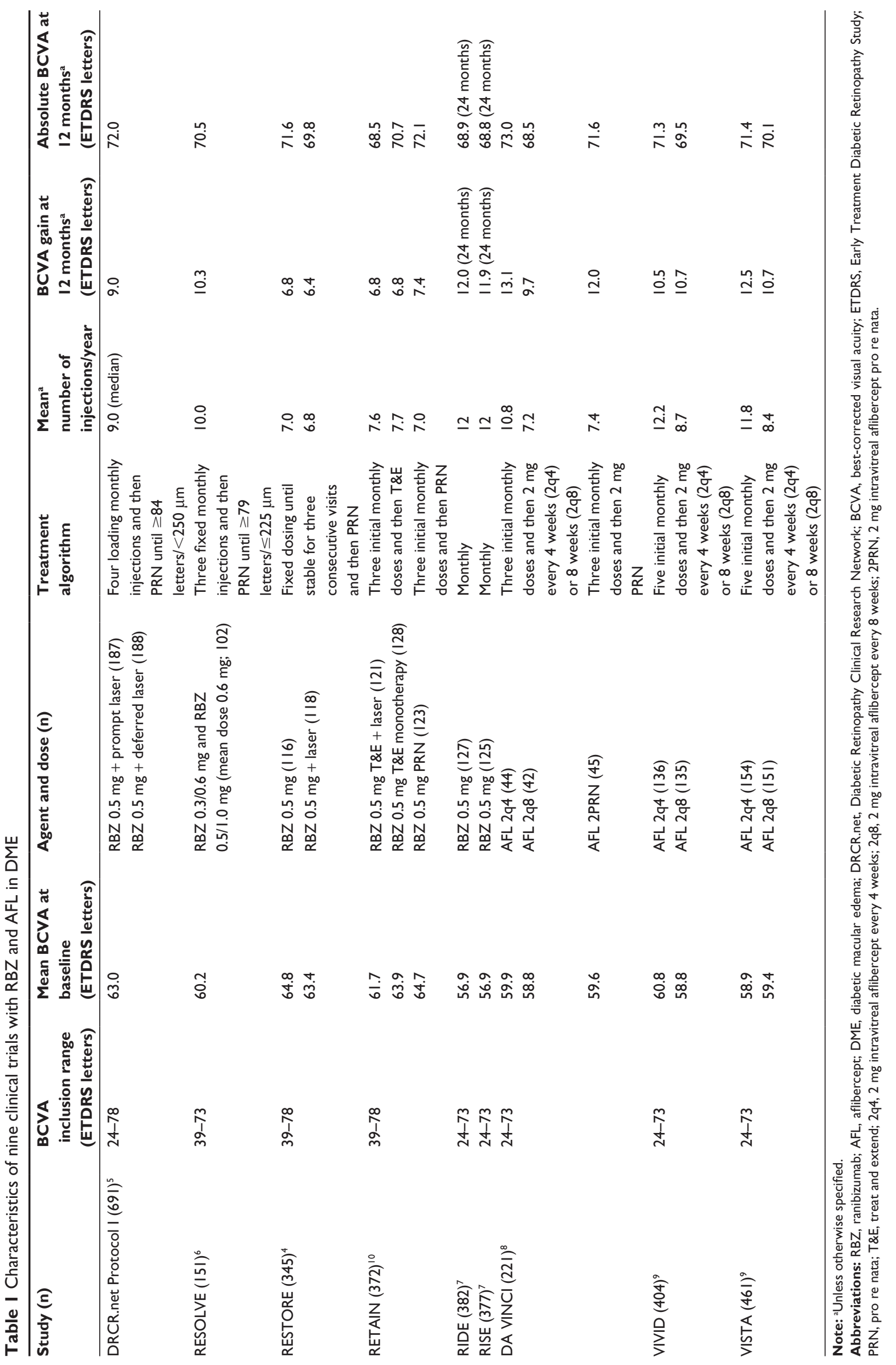


To corroborate this analysis, results from baseline BCVA-stratified analyses, which were performed with data obtained from RESTORE, RETAIN, and Protocol I, are also described here.

\section{Results}

\section{Cross-DME trial comparison}

The nine trials included 3,404 patients, of whom 1,616 were included in this analysis, with 707 treated with aflibercept $2 \mathrm{mg}$ and 909 treated with ranibizumab $0.5 \mathrm{mg}$ monotherapy (or with deferred laser) for at least 12 months (Table 1). The characteristics of each trial are provided in Table 1. They reveal wide variations in inclusion criteria translating into variations in mean BCVA at baseline.

Concurrently, mean BCVA gains after 12 months also varied considerably across trials. The greatest gain (13.1 letters) was observed for the DA VINCI trial of aflibercept $2 \mathrm{mg} \mathrm{q} 4$ (Figure 1). This trial enrolled patients with baseline VA in the range 24-73 letters, and this arm featured a mean BCVA of 59.9 letters at baseline.

The lowest gain (6.8 letters) using monotherapy was recorded for the RESTORE and RETAIN trials of ranibizumab $0.5 \mathrm{mg}$ for each of the PRN and treat and extend arms, respectively (Figure 1). The threshold for inclusion in these two trials was higher at 39 letters, and the maximum allowable baseline BCVA was also higher at 78 letters. With mean baseline BCVA of 64.8 and 63.9, these two trials also featured among the highest BCVA scores at baseline of all nine trials.

\section{Regression analysis}

While mean baseline BCVA levels varied from 56.9 letters to 64.8 letters, mean BCVA gains at month 12 or month 24 inversely varied from 13.1 letters to 6.8 letters. This inverse relationship was confirmed when baseline BCVA and BCVA gain were subjected to regression analysis, which revealed a significant, strong negative correlation between the two parameters ( $r=-0.85$ and $r^{2}=0.73, P<0.001$; Figure 2).

As a result of this correlation, a pattern emerges for mean absolute 12-month VA. The combined value of mean baseline BCVA plus mean gain in BCVA at 12 months, when examined across the nine trials and 14 anti-VEGF treatment arms studied, falls in a narrow range of 68.5-73.0 letters. The mean absolute VA achieved is thus relatively constant and very close to 70 letters irrespective of trial, drug, regimen, or number of injections (Figure 3).

\section{Stratified analysis}

BCVA baseline category analyses of RESTORE, RETAIN, and Protocol I support the findings of the present analysis at the individual patient data level. Analysis of RESTORE in DME has shown that baseline BCVA is a significant predictor of change in BCVA at 12 months and 36 months with ranibizumab (Figure 4). ${ }^{11}$ When patients in the RESTORE trial were categorized according to baseline BCVA, greater gains were evident for patients with $\leq 60$ letters at baseline versus those with $61-73$ letters and $>73$ letters of vision. ${ }^{4}$ In the group of patients receiving ranibizumab $0.5 \mathrm{mg}$ alone, the mean 12-month BCVA gains were 8.6 letters for patients

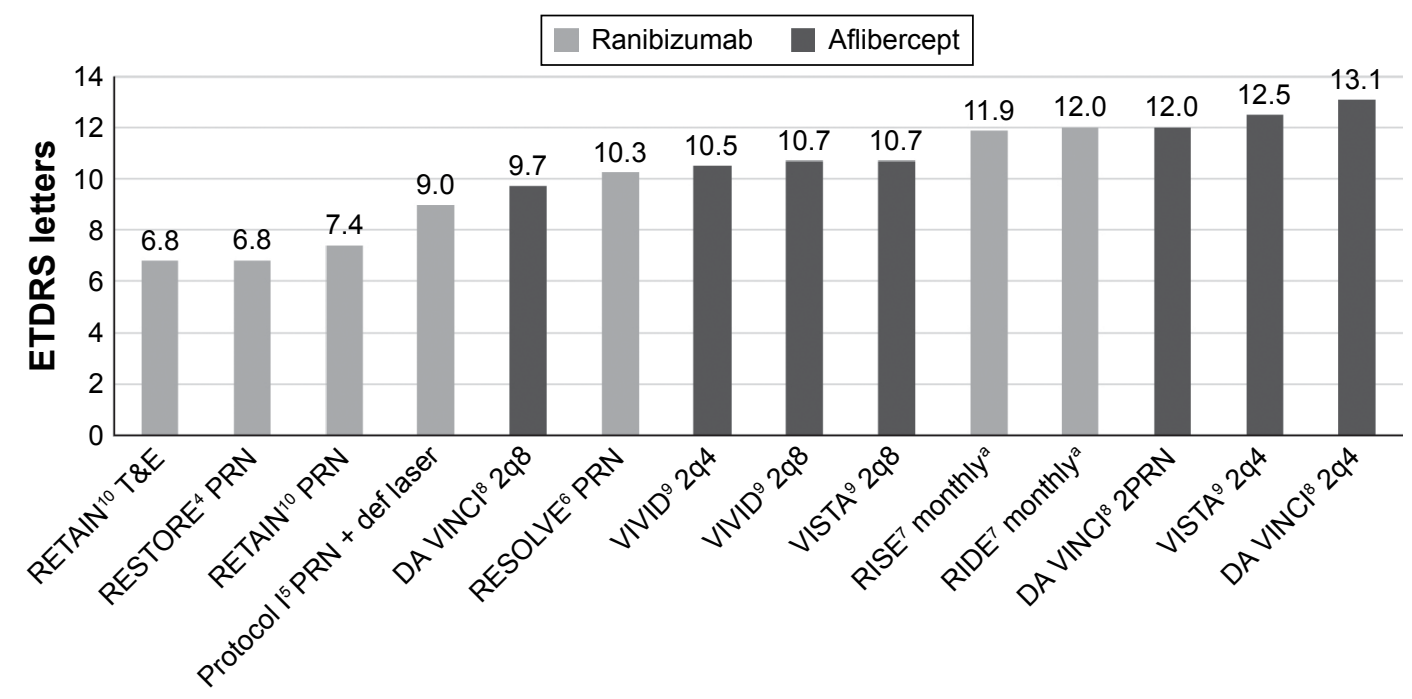

Figure I The mean BCVA gain across nine clinical trials of anti-VEGF agents in DME.

Note: ${ }^{2} 24-m o n t h$ data.

Abbreviations: BCVA, best-corrected visual acuity; VEGF, vascular endothelial growth factor; DME, diabetic macular edema; ETDRS, Early Treatment Diabetic Retinopathy Study; T\&E, treat and extend; PRN, pro re nata; def laser, deferred laser; 2q8, 2 mg intravitreal aflibercept every 8 weeks; $2 q 4,2$ mg intravitreal aflibercept every 4 weeks; 2PRN, $2 \mathrm{mg}$ intravitreal aflibercept pro re nata. 


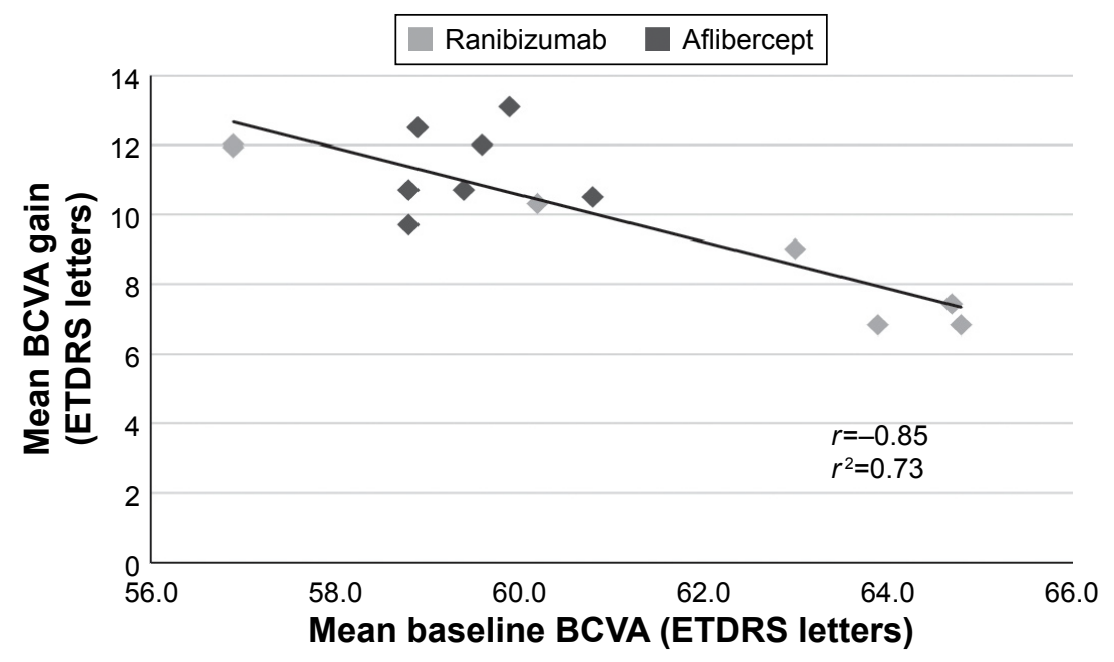

Figure 2 Regression analysis of mean BCVA gains versus mean baseline BCVA in the nine trials.

Abbreviations: BCVA, best-corrected visual acuity; ETDRS, Early Treatment Diabetic Retinopathy Study.

with $\leq 60$ letters of vision at baseline versus 7.5 letters for patients with 61-73 letters of vision at baseline and 2.1 letters for patients starting with $>73$ letters of vision (Figure 4).

Similar results were obtained in the RETAIN and Protocol I trials. In RETAIN, the group of patients with $\leq 60$ letters of vision at baseline gained 10.3 letters compared with 5.7 letters in the group with $>73$ letters of vision at baseline (data on file) (Figure 5). In a similar analysis of the Protocol I trial, patients were stratified according to baseline BCVA $\geq 66$ letters and $\leq 65$ letters. ${ }^{5}, 12$ In the group with worse vision at baseline, gains in BCVA were consistently greater than in the higher baseline BCVA group. In the ranibizumab plus deferred laser group (most analogous to ranibizumab monotherapy), patients in the worse baseline vision category gained $13 \pm 10$ letters at month 12 in contrast to $5 \pm 13$ letters in patients with better baseline vision. ${ }^{5}$

\section{Discussion}

The present analysis explores the effect of differences in baseline BCVA on the change in BCVA observed after $\geq 12$ months of anti-VEGF treatment across nine trials (14 arms) in patients with DME. The mean BCVA gains, which varied from 6.8 letters to 13.1 letters, were found to be negatively correlated to baseline BCVA, which inversely

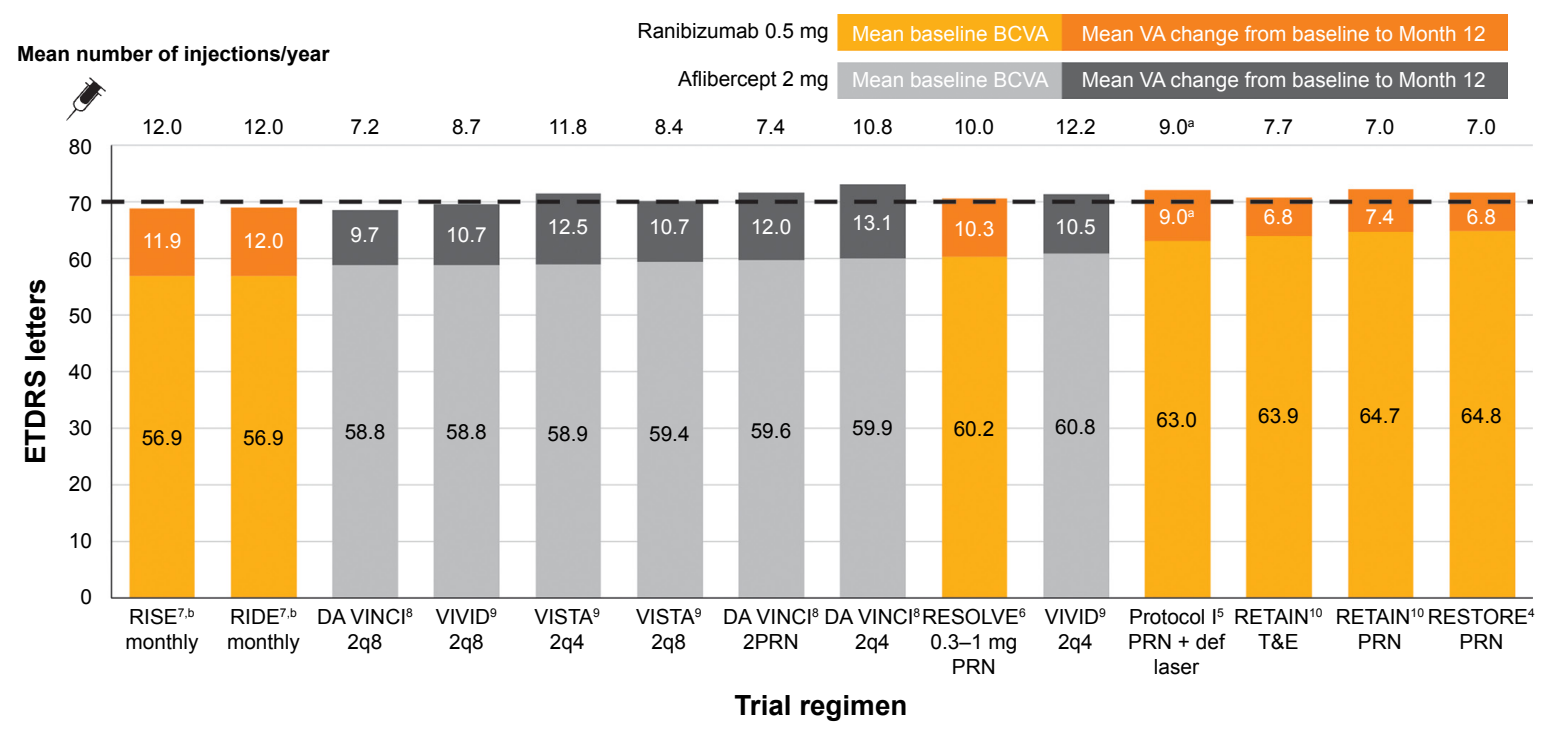

Figure 3 Baseline, gained, and final BCVA by trial and regimen.

Notes: aMedian. ${ }^{\mathrm{b}} 24-\mathrm{month}$ data.

Abbreviations: BCVA, best-corrected visual acuity; VA, visual acuity; ETDRS, Early Treatment Diabetic Retinopathy Study; 2q8, 2 mg intravitreal aflibercept every 8 weeks; $2 q 4,2 \mathrm{mg}$ intravitreal aflibercept every 4 weeks; 2 PRN, $2 \mathrm{mg}$ intravitreal aflibercept pro re nata; PRN, pro re nata; def laser, deferred laser; T\&E, treat and extend. 


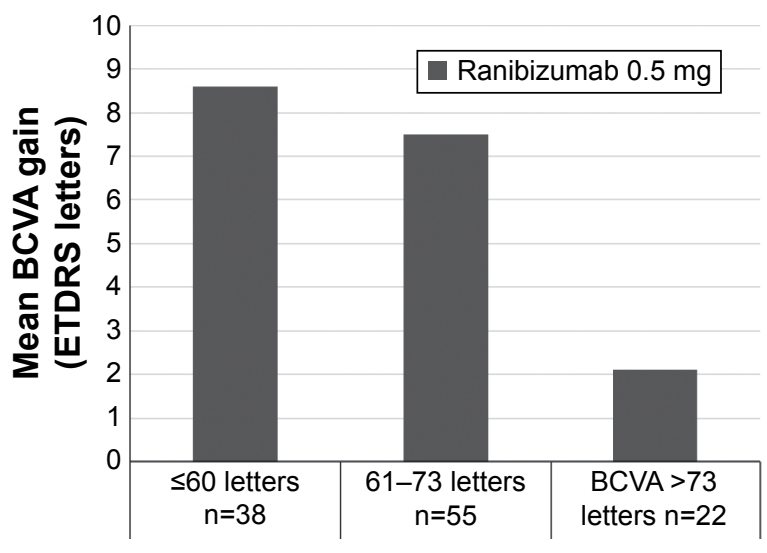

Figure 4 Mean change in BCVA from baseline to month 12 by baseline BCVA. Note: Data from RESTORE. ${ }^{4}$

Abbreviations: BCVA, best-corrected visual acuity; ETDRS, Early Treatment Diabetic Retinopathy Study.

varied from 64.8 letters to 56.9 letters. The results of the regression analysis demonstrated that mean BCVA at baseline could explain $73 \%$ of the variation of mean BCVA gain. The differences in gain seen across these trials may therefore be mainly due to differences in mean baseline BCVA, reflecting different inclusion criteria.

Category analyses performed in three of these trials (RESTORE, RETAIN, and Protocol I) further illustrate that anti-VEGF therapy results in higher BCVA gain in patients with poor vision. When the starting VA was better, antiVEGF agents did not provide such marked gains, although VA was still on average improved or at least maintained.

In addition, this analysis shows that irrespective of trials (and therefore drug, regimen, and number of injections), the mean absolute BCVA achieved at 12 months across patients in the studies is $\sim 70$ letters (20/40 Snellen VA equivalent). This result further challenges the notion that overall gain in BCVA from baseline is a measure of therapeutic success across trials with different inclusion criteria, in particular baseline BCVA range. Conducting cross-trial comparisons based on change in BCVA could therefore be misleading and should be done cautiously after adjustment for BCVA at baseline. ${ }^{13}$

However, the fact that the mean BCVA level at 1 year in many trials of ranibizumab and aflibercept in DME seems to be at a common level of 70 letters does not imply that there is a therapeutic ceiling in DME beyond which additional treatment is futile. Seventy letters is an average value. Patients who started with BCVA $>70$ letters were still able on average to gain up to more than five letters (Figures 4 and 5). In addition, it should be noted that $44.9 \%$ of patients treated with ranibizumab plus laser achieved a VA of $\geq 20 / 40$ (70 letters) after 1 year in RESTORE; after 3 years, the percentage of ranibizumab-treated patients who reached a VA of $\geq 20 / 40$ was $62.2 \%$ in RIDE, $63.2 \%$ in RISE, and $70 \%$ in Protocol I. ${ }^{14}$

This analysis has some limitations. Owing to the nature and source of the data, it was not possible to perform a multivariate analysis at the patient level across all trials on baseline characteristics such as age, HbA1c, blood pressure, duration of disease, and diabetes. However, a multivariate analysis of the RESTORE trial was conducted which, along with baseline BCVA, identified age and duration of DME and diabetes as predictors of responses to ranibizumab and laser therapy in patients with DME. ${ }^{11}$ Younger age and shorter diabetes duration were also associated with better VA outcomes and greater reduction of central subfield thickness following ranibizumab treatment in the Protocol I trial. ${ }^{12}$
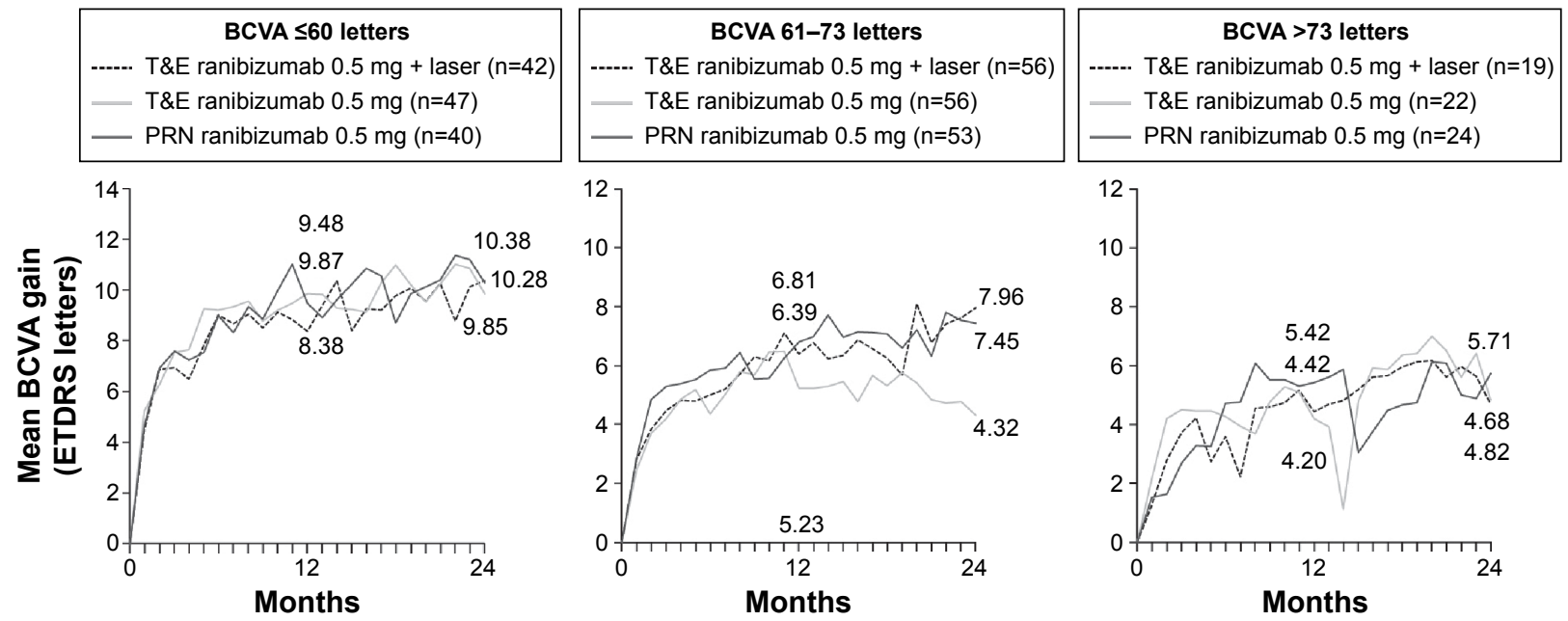

Figure 5 Mean change in BCVA from baseline to months 12 and 24 by baseline BCVA.

Note: Data from RETAIN (on file).

Abbreviations: BCVA, best-corrected visual acuity; ETDRS, Early Treatment Diabetic Retinopathy Study; T\&E, treat and extend; PRN, pro re nata. 
Diabetes tends to be very well controlled in clinical trials. This is in contrast to the situation encountered in clinical practice where patients with diabetes can be notoriously noncompliant. ${ }^{15}$ The mean absolute BCVA observed in these clinical trials may thus be different in clinical practice depending on baseline characteristics, such as duration of diabetes, duration of DME, and age. In common with diabetes control, other factors in clinical trials tend to be more controlled than in real-world practice by virtue of the inclusion and exclusion characteristics. Therefore, any analysis of baseline characteristics versus clinical outcomes will inevitably be hindered by a relatively small window of correlated data.

Nevertheless, the data presented here indicate that the current trials do not allow us to draw any conclusion about the comparative efficacy of the different anti-VEGF agents and the different regimens applied in DME. More head-to-head trials are needed to propose ideal regimen for anti-VEGF agents and develop guidelines.

In order to address this need, a recent study conducted by the DRCR.net, the protocol T, involving 660 subjects with DME and BCVA between 20/32 and 20/320, did propose as its primary objective to compare the efficacy and safety of intravitreal aflibercept, bevacizumab, and ranibizumab. ${ }^{16}$ This study, however, has limitations, as it is an open-label partly masked trial using a lower dose of ranibizumab $(0.3 \mathrm{mg})$ with a PRN regimen. This combination of dose and regimen is not approved in any jurisdiction, which raises some question as to its relevance to the approved regimen ex-US (0.5 mg PRN).

In the absence of more head-to-head randomized trials, new tools have been developed for comparative effectiveness research, addressing some of the limitations of cross-trial comparison highlighted in this analysis. ${ }^{13,17}$ The matchingadjusted indirect comparison model allows indirect comparisons of treatments and regimens to be performed across separate trials. ${ }^{17}$ It incorporates individual patient data from trials of one treatment to match baseline summary statistics reported from trials of another treatment. Treatment outcomes are then compared across balanced trial populations, thus providing comparative evidence more reliable than analyses based only on published aggregate data.

The fact remains that individualized regimens can be employed as means of lessening therapeutic and administrative burden. The commonly accepted approach of adopting individualized treatment regimens, with treatment intervals determined by the physician based on disease activity, as assessed by VA and/or anatomical parameters, is reflected in the current EU-SmPC for Lucentis ${ }^{\circledR}$ (Novartis International AG, Basel, Switzerland). ${ }^{18}$
Finally, the question as to why the mean BCVA observed in the trials included here seems to consistently even out at $\sim 70$ letters is worthy of further exploration. Of note, a similar plateau effect with anti-VEGF therapy has been reported in patients with neovascular age-related macular degeneration for whom a worse acuity at baseline was also found to predict more gain in vision. ${ }^{19}$ An inverse relationship between baseline BCVA and BCVA gain in neovascular age-related macular degeneration has also been observed in real-life clinical practice. ${ }^{20}$ Interestingly, patients in Protocol T were able to reach higher VA (mean VA of $\geq 76$ letters for the agents studied here) than what was observed in this analysis. ${ }^{16}$ This distinctive result suggests that other factors may play a role on final VA achievement; for instance, early treatment and different retreatment criteria may allow greater VA gain than those reached in previous trials.

It should also be noted that this plateau effect has been observed for the studied aflibercept and ranibizumab treatments in DME, and it is currently unclear whether or not it can be generalized to other anti-VEGF treatments and indications. In the BOLT study comparing repeated intravitreal bevacizumab to laser in patients with DME with mean baseline BCVA of 55.7 (+/-9.7) letters, the mean BCVA at 12 months was lower at $61.3( \pm 10.4)$, although other baseline characteristics might partly account for this result. ${ }^{21}$

The observational study LUMINOUS (NCT01318941) designed to observe the effectiveness and safety of ranibizumab, as well as the OCEAN study (NCT02194803) on ranibizumab treatment patterns and real-life ophthalmic monitoring, may help clarify the relevance of this plateau to routine clinical practice.

\section{Acknowledgments}

This work was presented previously at the 30th Asia-Pacific Academy of Ophthalmology Congress, Guangzhou, People's Republic of China, on April 1-4, 2015, and the 14th European School for Advanced Studies in Ophthalmology Congress, Istanbul, Turkey, on November 14-15, 2014. This study was supported by Novartis Pharma AG, Basel, Switzerland.

\section{Disclosure}

PUD is a consultant to Novartis, Alcon, Genentech, Alimera, Allergan, and Aerpio. JH has attended advisory board meetings, received travel grants and fees for presentations with Novartis, Bayer, Alimera, and Lutronic. SS has received research grants, travel grants, and attended advisory board meetings for Allergan, Bayer, Novartis, and Roche. JV is an employee of Novartis Pharma GmbH, Nürnberg, Germany. 
M-CM is an employee of Novartis Ireland Limited, Dublin, Ireland. AW, PM, and RH are employees of Novartis Pharma AG, Basel, Switzerland. PM is a board member of Novartis and Allergan and is a consultant for Novartis, Allergan, Alimera, and Bayer. The authors report no other conflicts of interest in this work.

\section{References}

1. American Diabetic Association. Standards of medical care in diabetes2012. Diabetes Care. 2012;35(suppl 1):S11-S63.

2. Fong DS, Aiello LP, Ferris FL 3rd, Klein R. Diabetic retinopathy. Diabetes Care. 2004;27(10):2540-2553.

3. Klein R, Klein BE, Moss SE, Davis MD, DeMets DL. The Wisconsin epidemiologic study of diabetic retinopathy. IV. Diabetic macular edema. Ophthalmology. 1984;91(12):1464-1474.

4. Mitchell P, Bandello F, Schmidt-Erfurth U, et al; RESTORE Study Group. The RESTORE study: ranibizumab monotherapy or combined with laser versus laser monotherapy for diabetic macular edema. Ophthalmology. 2011;118(4):615-625.

5. Elman MJ, Aiello LP, Beck RW, et al. Randomized trial evaluating ranibizumab plus prompt or deferred laser or triamcinolone plus prompt laser for diabetic macular edema. Ophthalmology. 2010;117(6): 1064.e-1077.e.

6. Massin P, Bandello F, Garweg JG, et al. Safety and efficacy of ranibizumab in diabetic macular edema (RESOLVE Study): a 12-month, randomized, controlled, double-masked, multicenter phase II study. Diabetes Care. 2010;33(11):2399-2405.

7. Nguyen QD, Brown DM, Marcus DM, et al; RISE and RIDE Research Group. Ranibizumab for diabetic macular edema: results from 2 phase III randomized trials: RISE and RIDE. Ophthalmology. 2012;119(4): 789-801.

8. Do DV, Nguyen QD, Boyer D, et al; DA VINCI Study Group. Oneyear outcomes of the DA VINCI Study of VEGF Trap-Eye in eyes with diabetic macular edema. Ophthalmology. 2012;119(8):1658-1665.

9. Korobelnik JF, Do DV, Schmidt-Erfurth U, et al. Intravitreal aflibercept for diabetic macular edema. Ophthalmology. 2014;121:2247-2254.

10. Prunte C, Fajnkuchen F, Mahmood S, et al; the RETAIN Study Group. Ranibizumab $0.5 \mathrm{mg}$ treat-and-extend regimen for diabetic macular oedema: the RETAIN study. Br J Ophthalmol. Epub 2015 Oct 9.
11. Mitchell P, Chong V. Baseline predictors of 3-year responses to ranibizumab and laser therapy in patients with visual impairment due to diabetic macular edema - The RESTORE study predictors analysis. ePoster presented at: 13th EURETINA Congress; September 26-29, 2013; Hamburg.

12. Bressler SB, Qin H, Beck RW, et al; Diabetic Retinopathy Clinical Research Network. Factors associated with changes in visual acuity and central subfield thickness at 1 year after treatment for diabetic macular edema with ranibizumab. Arch Ophthalmol. 2012;130(9):1153-1161.

13. Sivaprasad S, Fajnkuchen F, Régnier SA, Wright J, Larsen M. Using patient-level data for meaningful cross-trial comparisons in the visual impairment due to diabetic macular edema treated with anti-VEGF agents. Poster presented at: The Association for Research in Vision and Ophthalmology (ARVO) Meeting; May 3-7, 2015; Denver.

14. Boyer DS, Hopkins JJ, Sorof J, Ehrlich JS. Anti-vascular endothelial growth factor therapy for diabetic macular edema. Ther Adv Endocrinol Metab. 2013;4(6):151-169.

15. Marzec LN, Maddox TM. Medication adherence in patients with diabetes and dyslipidemia: associated factors and strategies for improvement. Curr Cardiol Rep. 2013;15(11):418.

16. Wells JA, Glassman AR, Ayala AR, et al. Aflibercept, bevacizumab, or ranibizumab for diabetic macular edema. N Engl J Med. 2015;372(13): 1193-1203.

17. Signorovitch JE, Sikirica V, Erder MH, et al. Matching-adjusted indirect comparisons: a new tool for timely comparative effectiveness research. Value Health. 2012;15(6):940-947.

18. European Medicines Agency [webpage on the Internet]. Lucentis: EPAR-Product Information. EMEA/H/C/000715-IB/0032 [updated November 13, 2015]. Available from: http://www.ema.europa.eu/ docs/en_GB/document_library/EPAR_-_Product_Information/ human/000715/WC500043546.pdf. Accessed February 1, 2016.

19. Finger RP, Wickremasinghe SS, Baird PN, Guymer RH. Predictors of anti-VEGF treatment response in neovascular age-related macular degeneration. Surv Ophthalmol. 2014;59(1):1-18.

20. Tufail A, Xing W, Johnston R, et al. The neovascular age-related macular degeneration database: multicenter study of 92976 ranibizumab injections: report 1: visual acuity. Ophthalmology. 2014;121(5): 1092-1101.

21. Michaelides M, Kaines A, Hamilton RD, et al. A prospective randomized trial of intravitreal bevacizumab or laser therapy in the management of diabetic macular edema (BOLT study) 12-month data: report 2. Ophthalmology. 2010;117(6):1078.e-1086.e.
Clinical Ophthalmology

\section{Publish your work in this journal}

Clinical Ophthalmology is an international, peer-reviewed journal covering all subspecialties within ophthalmology. Key topics include: Optometry; Visual science; Pharmacology and drug therapy in eye diseases; Basic Sciences; Primary and Secondary eye care; Patient Safety and Quality of Care Improvements. This journal is indexed on Submit your manuscript here: http://www.dovepress.com/clinical-ophthalmology-journal

\section{Dovepress}

PubMed Central and CAS, and is the official journal of The Society of Clinical Ophthalmology (SCO). The manuscript management system is completely online and includes a very quick and fair peer-review system, which is all easy to use. Visit http://www.dovepress.com/ testimonials.php to read real quotes from published authors. 